\title{
Teranóstico en medicina nuclear: ¿̇qué es y qué experiencia tenemos en Colombia?
}

\author{
Theranostic in nuclear medicine: what is it and what experience do \\ we have in Colombia?
}

\section{$\boldsymbol{\nu}$

\begin{tabular}{|l|}
\hline Palabras clave (DeCS) \\
\hline Nanomedicina \\
teranóstica \\
Medicina nuclear \\
Tomografía \\
computarizada por \\
tomografía de emisión \\
de positrones \\
Gammagrafía
\end{tabular}

Key words (MeSH)

Theranostic

vanomedicine

Positron emission

tomography computed

tomography

Radionuclide imaging
'Médica radióloga. Fellow de Medicina nuclear. Departamento de Medicina Nuclear, Hospital de la Universidad Nacional de Seúl, Corea del Sur.

2Médica(o) nuclear. Departamento de Imágenes Diagnósticas, Hospital Universitario Fundación Santa Fe de Bogotá, Colombia.

\section{Resumen}

En la era de la medicina personalizada y de precisión, enfocada en mejorar la atención en salud aprovechando al máximo las oportunidades que ofrecen los desarrollos biomédicos, tecnológicos, sociales y económicos de la actualidad, han aparecido nuevos términos como el de teranóstico. Este término nace de la fusión de los conceptos de terapia y diagnóstico y, aunque fue propuesto en años recientes, hace referencia a un abordaje que se ha utilizado desde hace mucho tiempo (1). El teranóstico consiste en una metodología donde el abordaje diagnóstico se hace enfocado hacia la intervención terapéutica individualizada, buscando proporcionar los mejores desenlaces para el paciente. El área de la medicina nuclear ha sido pionera en el teranóstico, pues el primer tratamiento basado en este concepto se realizó con yodo radiactivo ( ${ }^{131} \mid$ ) en pacientes con patología tiroidea. Actualmente, con los avances en imagen molecular e imágenes con genes reporteros (2), cada vez se encuentran disponibles más agentes teranósticos para proporcionar terapias individualizadas o "lesionalizadas", como se han empezado a llamar más recientemente (3). En la presente revisión se expone el abordaje teranóstico en medicina nuclear, enfatizando en el funcionamiento, las aplicaciones más frecuentes y la experiencia que se tiene en Colombia.

\section{Summary}

In the era of personalized and precision medicine, focused on improving health care by making the most of the opportunities offered by current biomedical, technological, social and economic developments, new terms such as theranostic have appeared. This term was born from the fusion of the concepts of therapy and diagnosis and, although it was proposed in recent years, it refers to an approach that has been used for a long time (1). Theranostic consists of a methodology where the diagnostic approach is focused on individualized therapeutic intervention, seeking to provide the best outcomes for the patient. The area of nuclear medicine has been a pioneer in theranostic, since the first treatment based on this concept was performed with radioactive iodine ${ }^{(131} \mid$ ) in patients with thyroid disease. Currently, with advances in molecular imaging and reporter gene imaging (2), more and more theranostic agents are available to provide individualized or "lesionalized" therapies, as they more recently have come to be called (3). In this review, the theranostic approach in nuclear medicine is exposed, emphasizing how it works, what are the most frequent applications and what experience we have in Colombia.

\section{Introducción}

Teranóstico es un término derivado de combinar las palabras terapia y diagnóstico, el cual se ha usado en muchos campos de la medicina para individualizar las terapias (4). Consiste en un abordaje diagnóstico enfocado a la intervención terapéutica, buscando proporcionar los mejores desenlaces para el paciente. Un ejemplo reconocido y utilizado hace muchos años es la detección de la sobreexpresión del oncogén HER2 en pacientes con cáncer de seno, para determinar si son candidatas a tratamiento con terapias anti-HER2 (lapatibnib o trastuzumab, por ejemplo), lo cual, en el escenario de neoadyuvancia, resulta en un mejor desenlace terapéutico con mayor tasa de respuesta patológica completa y mayor sobrevida libre de enfermedad (5).

\section{Teranóstico en medicina nuclear}

En medicina nuclear, teranóstico se refiere al uso de moléculas específicas (péptidos, agonistas, antagonistas, aminoácidos, análogos, etc.) que marcadas con un radionúclido específico permiten hacer diagnóstico y tratamiento de una enfermedad. Dichas moléculas marcadas con radionúclidos que emiten radiación y se utilizan para practicar estudios de imagen que permiten diagnosticar, localizar y/o estadificar una enfermedad; las mismas moléculas marcadas con un radionúclido que emite radiación $\alpha$ o $\beta$ van a tratar la enfermedad, es decir, las lesiones detectadas en el estudio diagnóstico $(4,6)$. De esta manera, se ve lo que se trata y se trata lo que se ve, que es el principio del teranóstico en medicina nuclear (figura 1). 


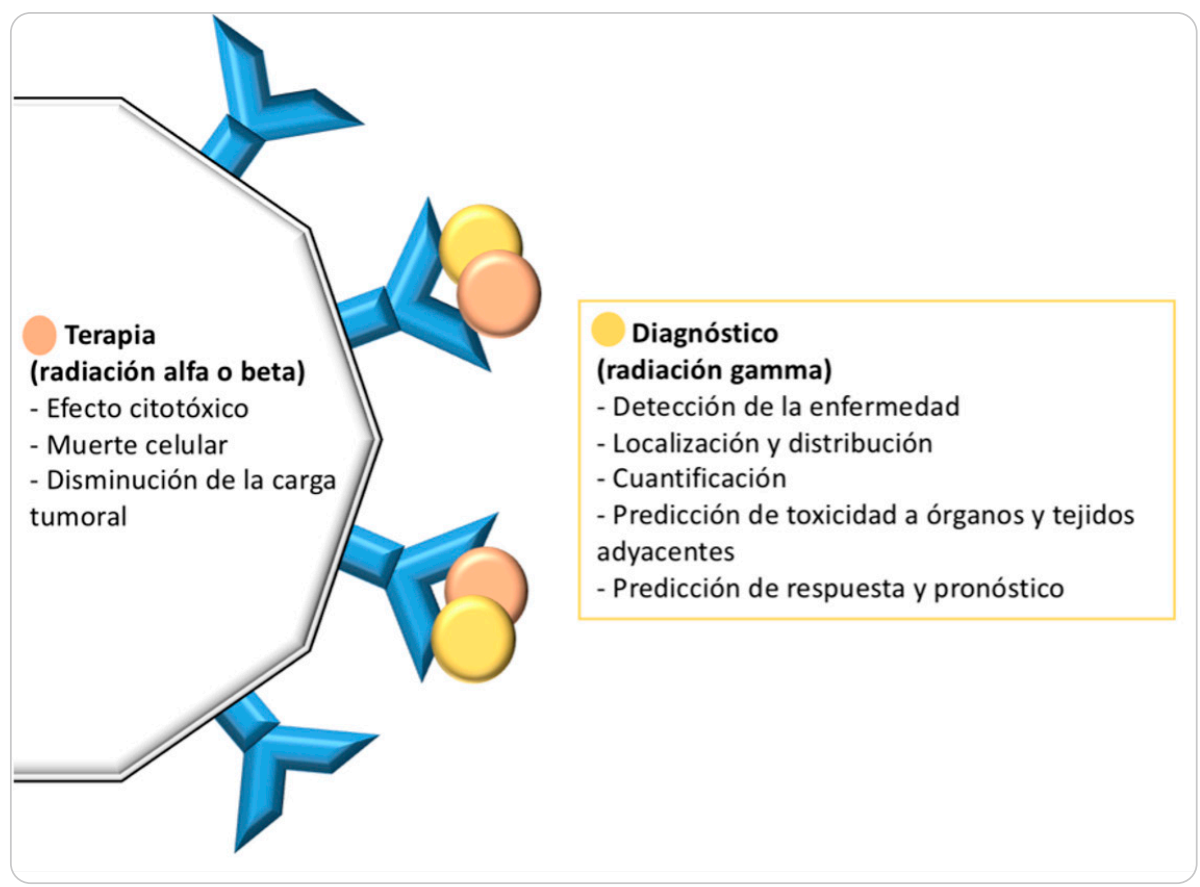

Figura 1. Principios del teranóstico en medicina nuclear. Se utiliza la misma molécula para hacer diagnóstico (marcada con un radionúclido con emisión $y$ ), y para tratamiento específico de la enfermedad (marcada con radionúclido que emite radiación $\beta \circ \alpha)$. a) Estudio diagnóstico

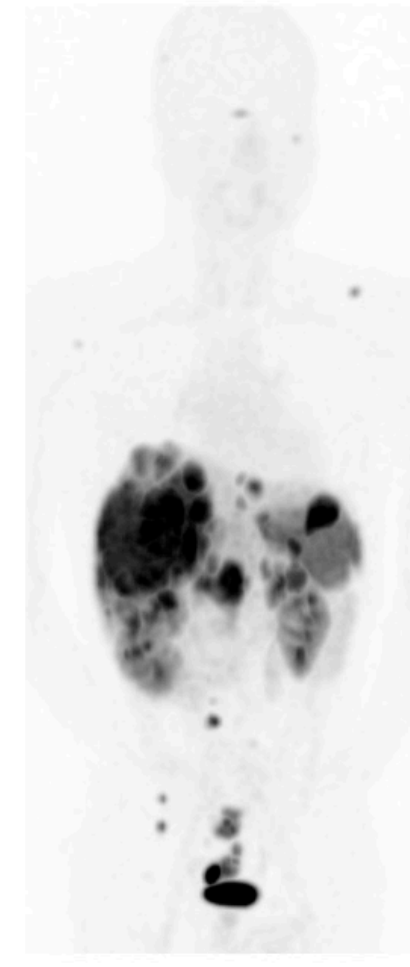

b) Seguimiento después de segundo ciclo

c) Seguimiento después de cuarto ciclo

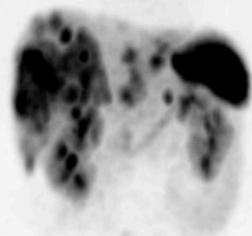

Lu-177 DOTATATE $200 \mathrm{mCi} \# 3-4$
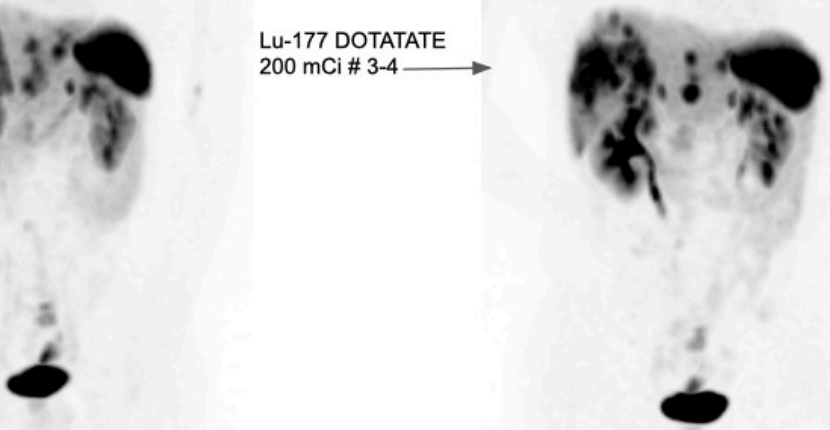

Figura 2. Paciente masculino de 54 años de edad con diagnóstico de tumor neuroendocrino rectal con metástasis hepáticas y óseas. a) Estudio diagnóstico: se confirmaron lesiones con receptores de somatostatina. Se administraron 2 ciclos de 177Lu, cada uno con dosis de $150 \mathrm{mCi}$. b) Control después del segundo ciclo: muestra resolución de las lesiones óseas y disminución del realce y tamaño de las lesiones hepáticas. Se administraron otros dos ciclos de 177Lu, cada uno con dosis de $200 \mathrm{mCi}$. c) Control después del cuarto ciclo: muestra disminución del realce y tamaño de la mayoría de las lesiones del lóbulo hepático derecho, con aumento de dos lesiones en el lóbulo hepático izquierdo. 
Aun cuando el término teranóstico es relativamente reciente, la técnica como tal se inició en medicina nuclear en 1941, cuando Saul Hertz administró la primera dosis de ${ }^{131}$ I para tratamiento de hipertiroidismo. Por muchos años el ${ }^{131}$ I fue el único ejemplo de un agente teranóstico, es decir, un radiotrazador capaz de tener los dos efectos: diagnóstico y terapéutico $(7,8)$. Este abordaje teranóstico se encuentra actualmente indicado en pacientes con cáncer diferenciado de tiroides en el escenario posquirúrgico, para: ablación de remanente de tejido tiroideo normal, tratamiento adyuvante en pacientes con sospecha o alto riesgo de enfermedad oculta, y tratamiento de enfermedad metastásica confirmada tanto locorregional como sistémica (9).

Posteriormente, en 1983, se desarrolló el ${ }^{131}$ I-MIBG (metayodobencilguanidina marcada con ${ }^{131} \mathrm{I}$ ) para diagnóstico (utilizando radiación $\gamma$ ) y terapia (utilizando radiación $\beta$ a dosis mucho mayores) de feocromocitoma y neuroblastoma. Actualmente, su uso se puede considerar parte del manejo sintomático paliativo en pacientes con enfermedad diseminada, inoperable o progresiva (10).

El siguiente desarrollo importante en este campo fue en el diagnóstico y tratamiento de tumores neuroendocrinos, especialmente gastroenteropancreáticos, los cuales, en su mayoría, expresan grandes cantidades de receptores de somatostatina. Para el abordaje diagnóstico se inició con el uso de ${ }^{111}$ In-pentetreotide, un análogo de la somatostatina radiomarcado utilizado para el diagnóstico gammagráfico (OcreoScan), posteriormente marcado con ${ }^{99 \mathrm{~m}} \mathrm{Tc}$ (11). Sin embargo, en 2000 emergieron nuevos radiofármacos para uso en PET y PET/TC $\left({ }^{68} \mathrm{Ga}\right.$-DOTATOC, ${ }^{68} \mathrm{Ga}$-DOTATATE, ${ }^{68} \mathrm{Ga}$-DOTANOC), con mejor resolución y calidad de imagen y mayor sensibilidad y especificidad, por lo que se consideran actualmente el método diagnóstico de elección (12-14). El abordaje terapéutico en estos pacientes está indicado y aprobado como segunda línea en caso de tumores metastásicos o inoperables con receptores de somatostatina positivos (demostrado por imágenes híbridas), bien diferenciados y moderadamente diferenciados (grado 1 y grado 2, según la clasificación de la OMS) (15). La terapia ha demostrado ser efectiva tanto con ${ }^{90} Y$-octreotate, como con ${ }^{177} \mathrm{Lu}$-octreotate, este último más ampliamente disponible y reconocido por los resultados del estudio NETTER-1, donde se demostraron como beneficios de su uso una mejoría sostenida en el estado de salud global de los pacientes, mayor sobrevida libre de síntomas y mayor tiempo de funcionalidad (16). Algunos autores recomiendan la terapia combinada con ${ }^{90} \mathrm{Y}$ y ${ }^{177} \mathrm{Lu}$, pero aún no se cuenta con estudios que soporten esta recomendación como definitiva (17) (figura 2).

El procedimiento teranóstico más reciente en medicina nuclear corresponde a los radiofármacos a base de antígeno prostático específico de membrana (PSMA, por sus siglas en inglés) marcados con ${ }^{68} \mathrm{Ga}$-PSMA o ${ }^{18} \mathrm{~F}$-PSMA para diagnóstico por PET/TC, lo cual ha revolucionado el diagnóstico del cáncer de próstata. El PSMA es una glicoproteína transmembrana que se expresa en las células prostáticas normales, pero que en las células neoplásicas se encuentra sobreexpresada, en relación directa con el grado tumoral, la agresividad de la enfermedad y el estadio patológico (18). El PSMA-PET/TC ha demostrado ser el estudio con mayor sensibilidad y especificidad en estos pacientes para detectar metástasis ganglionares y óseas en recaída bioquímica $(19,20)$. Actualmente, se está posicionando como herramienta de gran valor en la estatificación en pacientes con riesgo intermedio y alto, llevando a un cambio en el tratamiento hasta en el $12,6 \%$ de los casos
(21). El PSMA marcado con emisores $\beta$ o $\alpha$, permite realizar terapia y se constituye en una opción de tratamiento que cada vez gana mayor atención. Se han estudiado múltiples abordajes, y el ${ }^{177}$-Lu-PSMA y el ${ }^{225} \mathrm{Ac}$-PSMA son los que han mostrado mejores tasas de respuesta con menor toxicidad $(22,23)$, con disminución significativa de los niveles de PSA, buena tolerancia y mayor sobrevida, además de control de los síntomas (24).

\section{Teranóstico en Colombia}

En Colombia, el teranóstico inició de igual manera, con el uso del yodo radiactivo, que data de 1951, cuando se aplicaron las primeras terapias en el Instituto Nacional de Cancerología (INC). Actualmente es un procedimiento teranóstico que se realiza de manera rutinaria prácticamente en cualquier servicio de medicina nuclear del país.

En el campo de los tumores neuroendocrinos, los estudios diagnósticos iniciaron con análogos de somatostatina marcados con ${ }^{111}$ In y ${ }^{99 \mathrm{~m}} \mathrm{Tc}$, y actualmente se cuenta con ${ }^{68} \mathrm{Ga}$-DOTA PET/TC, el cual tiene mayor precisión y es el estudio de elección para el diagnóstico de estos pacientes. El uso terapéutico con ${ }^{177} \mathrm{Lu}$-DOTA se inició en 2009 y se realiza cada vez de manera más frecuente en algunas instituciones.

Recientemente, en 2019, se inició el trabajo con ${ }^{68} \mathrm{Ga}$-PSMA y en 2020 con ${ }^{18} \mathrm{~F}$-PSMA para diagnóstico de cáncer de próstata, implementado ahora con mayor frecuencia por algunas instituciones. La terapia con ${ }^{177} \mathrm{Lu}$-PSMA, como opción de tratamiento para pacientes con cáncer de próstata metastásico, se inició a finales de 2020.

\section{Conclusión}

El abordaje teranóstico en medicina nuclear tien múltiples ventajas. Hacer un diagnóstico preciso dirigido al tratamiento, permite seleccionar adecuadamente los pacientes para recibir dicho tratamiento, con lo cual se tiene además la posibilidad de predecir directamente la respuesta al mismo y determinar el pronóstico (25). Esto hace que el teranóstico tenga un papel definitivo en la medicina actual, y es fundamental que los clínicos conozcan estas opciones terapéuticas y su disponibilidad en el país, para que cada vez se pueda hacer más y mejor uso de las mismas.

\section{Referencias}

1. Hermann K, Larson SM, Weber W. Theranostic concept: More than just a fashion trend-Introduction and Overview. J Nuc Med. 2017;58(supplement 2):1S-2S.

2. Kang JH, Chung JK. Molecular-Genetic imaging based on reporter gene expression. J Nucl Med. 2008;49:164S-79S.

3. Chung JK, Kim MJ, Youn H. Lesionalized therapy beyond personalized therapy in cancer management. J Korean Med Sci. 2014;29(10):1331-2.

4. Gomez JF, Nunes RE, Coutinho A, Zaniboni E, Costa LB, Barbosa FG, et al. Theranostics in nuclear medicine: Emerging and re-emerging integrated imaging and therapies in the era of precision oncology. RadioGraphics. 2020;40:1715-40.

5. Vbric S, Pejcic I, Filipovic S, Kocic B, Vbric M. Current and future anti-HER2 therapy in breast cancer. J Buon. 2013;18(1):4-16.

6. Marcu L, Bezak E, Allen B. Global comparison of targeted alpha vs targeted beta therapy for cancer; in vitro and in vivo clinical trials. Crit Rev Oncol/Hematol. 2018;123:7-20.

7. Choudhury PS, Gupta M. Differentiated thyroid cancer theranostics: radioiodine and beyond. Brit J Radiology. 2018;91:1091.

8. Silberstein EB. Radioiodine: the classic theranostic agent. Semm Nucl Med. 2012;42(3):164-70.

9. Haugen BR, Alexander EK, Bible KC, et al. 2015 American thyroid association management guidelines for adult patients with thyroid nodules and differentiated thyroid cancer: The American Thyroid Association Guidelines Task Force. Thyroid. 2016;26(1):1-133. 
10. Kotecka-Blicharz A, Hasse-Lazar K, Handkiewicz-Junak D, Gawlik T, Pawlaczek A, Oczko-Wojciechowska M, et al. 131-I MIBG therapy of malignant pheochromocytoma and paraganglioma tumours - a single-centre study. Endokrynol Pol. 2018;69(3):246-51.

11. Krenning EP, Bakker WH, Breeman WA, et al. Localisation of endocrine-related tumors with radioiodinated analogue of somatostatin. Lancet. 1989;1(8632):242-4.

12. Singh S, Poon R, Wong R, Metser U. 68Ga PET Imaging in patients with neuroendocrine tumors: A systematic review and meta-analysis. Clin Nucl Med. 2018;43(11):802-10.

13. Levine R, Krenning EP. Clinical history of the theranostic radionuclide approach to neuroendocrine tumors and other types of cancer: historical review based on an interview of Eric P. Krenning by Rachel Levine. J Nucl Med. 2017;58(supp1 2):3S-9S.

14. Bodei L, Mueller-Brand J, Baum RP, et al. The joint IAEA, EANM, and SNMMI practical guidance on peptide receptor radionuclide therapy (PRRNT) in neuroendocrine tumours. Eur J Nucl Med Mol Imaging. 2013;40(5):800-16.

15. Rindi G. The ENETS guidelines: the new TNM classifica- tion system. Tumori. 2010;96(5):806-9.

16. Strosberg J, Wolin E, Chasen B, et al. Health-related quality of life in patients with progressive midgut neuroendocrine tumors treated with 177Lu-Dotatate in the Phase III NETTER-1 Trial. J Clin Oncol. 2018;36(25):2578-84.

17. Cremonesi M, Ferrari M, Bodei L, Tosi G, Paganelli G. Dosimetry in peptide radionuclide receptor therapy: a review. J Nucl Med. 2006;47(9):1467-75.

18. Hofman MS, Lawrentschuk N, Francis R, Tang C, Vela I, et al. Prostate-specific membrane antigen $\mathrm{PET} / \mathrm{CT}$ in patients with high-risk prostate cancer before curativeintent surgery or radiotherapy: a prospective, randomised, multicentric study. Lancet. 2020;395(10231):1208-16.

19. Perea M, Papa N, Roberts M, Williams M, Udovicih C, Vela I, et al. Gallium-68 prostate-specific membrane antigen positrón emission tomography in advanced prostate cáncer-Update diagnostic utility, sensitivity, specificity, and distribution of prostatespecific membrane antigen-avid lesions: A systematic review and Meta-analysis. European Urol. 2020;77:403-17.

20. Hofman MS, Violet J, Hicks RJ, et al. [177Lu]-PSMA-617 radionuclide treatment in patients with metastatic castration- resistant prostate cancer (LuPSMA trial): a single-centre, single arm, phase 2 study. Lancet Oncol. 2018;19(6):825-33.

21. Van Kalmthout LWM, van Melick HHE, Lavalaye J, Meijer R, Kooistar A, de Klerk $\mathrm{JMH}$, et al. Prospective validation of Gallium- 68 prostate specific membrane antigenpositron emission tomography/computerized tomography for primary staging of prostate cancer. J Urol. 2020;203:537-45.

22. Wu H, Xu T, Wang X, Yu Y-B, Fan Z-Y, Li D-X, et al. Diagnostic performance og 68Gallium labelled prostate-specific membrane antigen positrón emission tomography/ computed tomography and magnetic resonance imaging for staging the prostate cáncer with intermediate or high riesk prior radical prostatectomy: a systematic review and Meta-analysis. World J Mens Health. 2020;38(2):208-19.

23. Calopedos RJS, Chalasani V, Asher R, Emmett L, Woo HH. Lutetium-177-labelled anti-prostate-specific mem- brane antigen antibody and ligands for the treatment of metastatic castrate-resistant prostate cancer: a systematic review and meta-analysis. Prostate Cancer Prostatic Dis. 2017;20(3):352-60.

24. Ahmadzadehfar $\mathrm{H}$, Wegen $\mathrm{S}$, Yordanova A, et al. Overall survival and response pattern of castration-resistant meta- static prostate cancer to multiple cycles of radioligand therapy using [177Lu]Lu-PSMA-617. Eur J Nucl Med Mol Imaging. 2017;44(9):1448-54.

25. Yordanova A, Eppard E, Kurping S, et al. Theranostics in nuclear medicine practice. OncoTargets Ther. 2017;10:4821-8.

\section{Correspondencia}

Vanessa Murad

158 Chang Gyung Gung-Ro, Jongno-gu,

Seoul, Corea

murad.vanessa@gmail.com

Recibido para evaluación: 10 de enero de 2021

Aceptado para publicación: 21 de abril de 2021 